\title{
Aproximación al "esquema L" de Lacan y sus implica- ciones en la clínica (parte I).
}

What the "schema L" consists of? (Part I).

\author{
José Manuel García Arroyo a , María Luisa Domínguez López ${ }^{\text {b }}$
}

${ }^{a}$ Médico Psiquiatra. Profesor Asociado. Departamento de Psiquiatría de la Facultad de Medicina de Sevi-

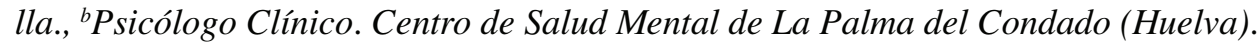

Correspondencia: José Manuel García Arroyo (jmgarroyo@us.es)

Recibido: 13/02/2010; aceptado: 15/07/2010

RESUMEN: A lo largo de la obra de Lacan es común encontrar diferentes diagramas que pueden entenderse como intentos de formalizar ciertos aspectos de la teoría psicoanalítica; estos últimos deben quedar claros, al menos desde el punto de vista teórico, para poder actuar correctamente en el trabajo analítico. En esta ocasión, vamos a centrarnos en uno de ellos: el "esquema L", cuya presentación se produce en uno de sus seminarios orales, dedicado a la posición del "yo" en la teoría de Freud y que captó la atención de facultativos y especialistas de aquella época. En él su autor trabaja, de un modo ciertamente original, el funcionamiento de las relaciones intersubjetivas en sus conexiones con el lenguaje. En la primera parte del trabajo, vamos a estudiar los componentes del "esquema L" y las dos relaciones fundamentales que se establecen entre ellos y que forman los ejes "imaginario" y "simbólico". Lacan los considera en oposición pues se interfieren mutuamente, de modo tal que el mensaje inconsciente queda interrumpido o cortocircuitado constantemente por la relación imaginaria, formada por el "yo" y su semejante, el "otro".

PALABRAS CLAVE. Esquema L. Yo. Sujeto. Topología. Simbólico. Imaginario.
ABSTRACT: Along Lacan's work it's usual to find several diagrams that can be understood as an attempt to conceptualize some aspects of the psychoanalytic theory. The latter ones must be clear, at least from the theoretical point of view, to be able to a properly psychoanalytic work. At this time, we are going to centred on one of them: schema L, whose presentation take place at one of Lacan's seminar about the ego position in Freud's theory and which catch the eye of specialist of that time. It's also an original way of expound the performance of the inter- subjective relations in its connections with the language. In the first part of this work we study the components of the "schema L" and two of the main relations among themselves that form the two axis: "imaginary" and "symbolic". Lacan consider these to be in opposition since they interfere with each other so that the unconscious message is broken by the imaginary relation between the ego and its equal "the other".

KEY WORDS: Schema L. Ego. Subject. Topology. Symbolic. Imaginary.

\section{Introducción}

En el curso 1.954-55 Lacan se dedicó a esclarecer la posición del "yo", durante el seminario impartido periódicamente en el anfiteatro del hospital de Sainte Anne, que le había cedido el profesor Jean Delay. Este seminario, que gozó de gran concurrencia y popularidad, llevaba el título de: El yo en la teoría de Freud y en la técnica psicoanalítica (1). En un momento avanzado del mismo, concretamente en la clase 
XIX, su autor propone una especie de gráfico que va a ocupar un lugar privilegiado en su enseñanza por unos pocos años más y al que da el nombre de "esquema L" debido a que la forma que muestra recuerda a la letra "lambda" del alfabeto griego.

Los diversos esquemas, que comenzaron a aparecer en los textos de este gran psiquiatra y psicoanalista parisino en la década de 1.950, son intentos de formalizar por medio de diagramas, ciertos aspectos de la teoría psicoanalítica y ese es el caso del que ahora ocupa nuestra atención. En este sentido, no podemos considerar al "esquema L" un modelo, sino más bien un material explícito con el que se pretende fijar un conjunto de ideas, que han de estar claras para poder ubicarse al lado del paciente y efectuar un verdadero tratamiento analítico. De ahí que, a pesar de su sencillez y elegancia, funcione como un condensador de información pues ilustra asuntos de sumo interés, como son: las relaciones que establecemos con nuestros semejantes, la barrera que supone el "yo" en la cura y en el acercamiento a uno mismo, la forma en la que el sujeto se (auto) representa, las conexiones que se establecen entre el "yo" y el inconsciente, el lugar del lenguaje en este último diferenciando entre tipos de discurso, etc.

Además de lo expuesto, el tema al que dedicamos este artículo representa la primera incursión de Lacan en el complejo pero fascinante mundo de la "topología". Consiste esta última en aquel dominio de las matemáticas en el que se interpreta la geometría de un modo particular: considerando solo la posición respectiva de los elementos de una figura, con exclusión de cualquier noción de magnitud espacial o de unidad de medida (2). Esta incursión en el dominio de las ciencias exactas va a ocupar la enseñanza lacaniana, de un modo ciertamente original, a partir de la década de los 70 (3). En esta línea, el "esquema L" implica una representación espacial de relaciones, no tratándose de espacio físico, sino "topológico", donde no importan las distancias entre los componentes, sino más bien las conexiones que se establecen, como es el caso de: sucesión, secuencia, continuidad, interposición, vecindad, etc (4) (nota 1).

Añadimos que el esquema al que nos referimos muestra semejanzas con algunos gráficos que el antropólogo Lévi-Strauss, amigo personal de Lacan, presentó en dos de sus textos mas conocidos y que le sirvieron a este último como punto de referencia $(5,6)$.

En su constitución, diremos que está formado por una serie de puntos conectados entre sí por medio de vectores. Cada punto es designado por un signo del álgebra lacaniana (a, S, A,...) y que, con el paso del tiempo, su inventor va a denominar "matemas", debido a la semejanza que tienen dichos signos con el lenguaje matemático (nota 2). En cambio, los vectores están destinados a poner de manifiesto la relación estructural existente entre los signos y la dirección que siguen dichas conexiones. Es posible, en este contexto, establecer recorridos que dan cuenta de cómo se producen las relaciones entre los distintos elementos que lo componen. 
ORIGINALES Y REVISIONES

De esta forma, al posicionar los diferentes componentes en los lugares vacíos de esquema y establecer trayectorias a seguir, el conjunto puede entenderse como un mapa de las relaciones intersubjetivas, que Lacan llega incluso a aplicar en su "cuarto seminario" (4) a los diferentes contactos interpersonales que se establecen en el caso Dora y en el de la joven homosexual, ambos estudiados por Freud (7, 8). Además, permite dar cuenta de una correcta concepción de la experiencia analítica, en tanto experiencia intersubjetiva, contraviniendo así la postura basada en las "relaciones de objeto", tan distantes a la práctica freudiana. Quiere decir que se desmenuzan, de una forma sencilla y clara, diferentes incidencias que pueden producirse durante el tratamiento y cuál ha de ser el lugar que el analista debe ocupar durante el desarrollo del mismo.

En las páginas que siguen vamos a tratar de esclarecer cuáles son los componentes, el funcionamiento y las lecturas posibles del "esquema L" que, aunque parezca bastante complicado, no lo es en absoluto. Añadimos que, cuando quede aclarado, podrá descubrirse la gran utilidad que tiene en la práctica clínica y la potencialidad que posee al orientar el proceso de la cura. Para entenderlo mejor, iremos construyéndolo progresivamente, colocando cada uno de sus componentes, para analizar después las direcciones que toma y sus diferentes significaciones. Con el fin de comprobar su utilidad práctica, ilustraremos la exposición con numerosos casos clínicos tomados de nuestra práctica diaria.

\section{Componentes del "esquema L".}

La composición del "esquema L" es “tetrádica", lo que significa que tiene cuatro elementos, dándose la particularidad de que cada uno de ellos es abordable en función de las relaciones que establece con los restantes (8). Comenzaremos por enumerarlos y ver cómo se conectan entre sí:

2.1. El "yo". En un trabajo previo expusimos las características de esta instancia en consonancia con una postura freudiana (10). En esa misma línea, diremos que el "yo" consiste en aquel lugar donde se intenta producir la representación propia sin fallas ni fisuras, lo que indica que el ego se encuentra prisionero de la ilusión de "unidad" y "síntesis". Añádase, que la organización yoica deja fuera de su perímetro todo aquello que no resulta coherente con sus pretensiones pero, como sabemos, todo lo arrojado fuera no desaparece espontáneamente sino que vuelve de alguna manera. De ahí se desprende, que en su constitución muestre una base narcisista (10).

El "yo" se vertebra sobre creencias acerca de la realidad y de uno mismo, que no tienen por qué casar necesariamente con ninguno de ellos, de donde se sigue que se estructura sobre el (auto) engaño y el espejismo. 
Se representa en el esquema con la letra (a), de la voz francesa "autré" y que puede traducirse como "otro".

2.2. El "otro". Se trata del semejante, el "alter-ego", y se expresa con el matema (a'). En el esquema lacaniano ambos elementos son intercambiables, pudiéndose afirmar que "el yo es otro" y "el otro es yo" o, como dijo Rimbaud: "Je est un autre". De ahí que la letra "a" ("autre") se emplee en ambos casos.

Este aserto puede demostrarse fácilmente, ya que:

-Por un lado, la primera de las fórmulas ("el yo es otro") tiene que ver con los fenómenos de "proyección", en las múltiples formas en que se puede presentar en las relaciones cotidianas: colocar los defectos propios en los demás, ser crítico o exigente con los semejantes como se es consigo mismo, no aceptar a los otros cuando uno no se acepta, hallarse siempre pendiente de la aprobación o el reconocimiento ajenos para sentirse aprobado e importante, etc.

-Por otro, la segunda fórmula ("el otro es yo") especifica de modo claro los sucesos propios de la "identificación” que es, según Freud, el mecanismo sustancial en la formación del "yo". Efectivamente, este último puede entenderse como una suma de identificaciones, aspecto que puede descubrirse fácilmente en las sesiones con los pacientes (10) (nota 3 ).

Lacan ha mostrado que el origen del "yo" se encuentra en el exterior, dado que se organiza a partir de la imagen que se produce en el espejo, donde se halla a un "otro" reflejado. De ahí la alienación fundamental sobre la que se organiza dicha estructura y que marcará el destino posterior de su portador $(11,12)$.

Dando un paso más diremos que el "yo", en los contactos narcisistas que le son propios, mantiene una confusión con el semejante. Esto puede recogerse con facilidad en nuestros consultorios donde, muchas veces, no es fácil saber de quién habla el paciente, si de sí mismo o de otra persona. Así, una de nuestras consultantes, bastante envidiosa por cierto, cuando habla de una amiga indica que es una persona que "envidia a todo el mundo" y "se la come la envidia" y, las más de las veces, no es fácil deducir, a partir de sus enunciados, a quién se refiere en realidad pues se ve cómo sus propios sentimientos están "depositados” en esta última.

De esta manera, se rebela la falacia implícita en la idea del "yo autónomo" de Hartmann (13), dado que el ego no puede considerarse independiente de las relaciones con quiénes lo rodean; se trataría de una ilusión de "autodominio" y "autocontrol", que queda refutada constantemente por la clínica.

2.3. La relación "yo-otro". Existe una conexión directa entre estos dos elementos analizados, que se expresa con el emparejamiento del "yo" al mundo simétrico del "otro"; nos hallamos en el plano del espejo y que se representa como (a-a')

A esta relación que ahora describimos, Lacan la denomina "eje imaginario", debido a que el ego es básicamente una construcción imaginaria, ya que su carácter fundamental es de espejismo. Aquí es donde debemos ubicar el inmenso poder que 
ORIGINALES Y REVISIONES

tienen las imágenes sobre el humano, pudiéndose observar en todo su esplendor en las relaciones sociales, las cuáles se hallan tejidas sobre aquellas, funcionando del siguiente modo: "me presento ante ti como una persona maravillosa, para que tú me veas de esa misma forma; paralelamente, te veo a ti extraordinario, porque quieres producir en mí esa idea. Conclusión: "los dos somos estupendos y maravillosos" (nota 4).

Los "mecanismos de defensa", de sobra conocidos por todos, se hallan ligados a esta conexión dual de carácter narcisista, dado que con ellos se pretende obtener la propia aceptación y la ajena. Con ellos se procura que el "yo" resulte "absuelto" de los aspectos perturbadores que distorsionan la imagen impoluta que quiere conservar y que, obviamente, desea producir en los demás. Podemos suponer, en este sentido y a título de ejemplo, que "racionalizar" implica presentar explicaciones coherentes desde el punto de vista lógico, pero incorrectas, sobre el propio comportamiento para sentirse lo más adecuado posible o ubicarse ante los demás como alguien "sin tachas". Análogamente, "fantasear" indica deformar las circunstancias que se viven hasta hacerlas admirables para elevar la autoimportancia, el nivel de las propias ejecuciones y/o deformar aquellos encuentros con los demás que hayan resultado frustrantes. Así podríamos seguir con todas y cada una de las defensas que se han descrito. Como puede verse, estos recursos mentales no son entidades independientes de la relación intersubjetiva, tal como fueron enunciados por Anna Freud (14).

También se han de ubicar en este eje las "identificaciones" pues, al ponernos en contacto con los demás, encontramos similitudes y nos identificamos con ellos. Como ejemplo, presentamos a una paciente nuestra quién, tras varias rupturas sentimentales, hace una elaboración paranoide de la figura masculina, al tiempo que busca a interlocutoras que le den la razón. Estas últimas son, por lo general, mujeres que están pasando por circunstancias parecidas y con las que la paciente puede identificarse. El proceso seguido por ella da cuenta de la estricta reciprocidad del "yo" con el "otro", en cuyo caso no es extraño que se produzca un discurso común, monótono y repetitivo (p. ej. "los hombres nos hacen daño", "hay que tener mucho cuidado con ellos", "nosotras somos débiles y tenemos que protegernos", etc). Las mismas leyes expuestas siguen la pertenencia a organizaciones comunes (equipo de fútbol, partido político, profesión,...) cuyos miembros, identificándose mutuamente, articulan una producción verbal común con idénticas claves (significantes).

El eje (a-a') recibe también el nombre de "muro del lenguaje", debido a que el discurso que se produce en su seno es un impedimento para acceder al verdadero conocimiento. El discurso común, al que Lacan llama en otro lugar "palabra vacía" (15), sigue estas características pues sirve para la reconfirmación de uno en el otro y viceversa; se trata de un lenguaje que participa del engaño más absoluto, de ahí el nombre que se le ha dado. Encontramos aquí el lugar propio de las "pamplinas sociales", como son: destacar, ser el mejor, no quedar por debajo, presumir, tener el 
mejor empleo, la mejor familia, mentir, etc. Esto mismo hace que la relación social pueda entenderse como un "diálogo de sordos", lo que indica que cuando la gente se comunica, ese lenguaje común siempre se halla mediatizado por el imaginario que supone que un "yo" se comunica con otro "yo" distinto, pero semejante a él.

Por lo tanto, si hay algo que conocer evidentemente se hallará más allá del "eje imaginario", del "muro del lenguaje" que, como vemos, procura el desconocimiento más absoluto (ver figura 1).

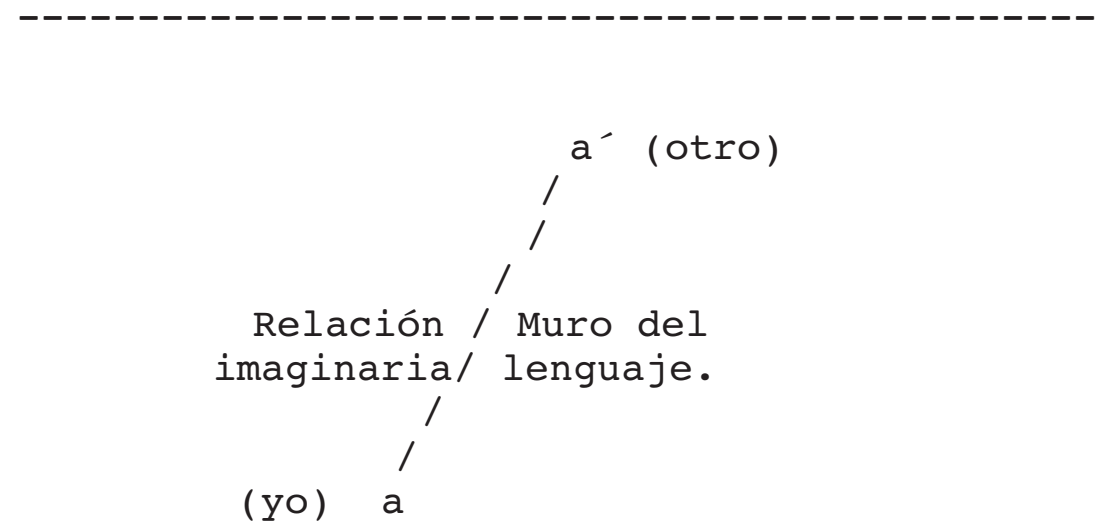

Figura 1 .

Añadimos que la relación imaginaria se pondrá de manifiesto de un modo particular en cada una de las diferentes estructuras clínicas, como es el caso de: la búsqueda constante de aprobación de la histérica, la retracción social del fóbico debido al gran temor al rechazo que muestra o, incluso, se halla en el origen del control que mantiene al obsesivo tan alerta para que nada de lo que suceda a su alrededor se le escape.

2.4. El sujeto. Lacan se esfuerza en diferenciar el "yo" del "sujeto", ya que frecuentemente se confunden en la literatura al uso. Para Freud la realidad del sujeto se halla en el inconsciente, excluido el sistema del "yo"; por tanto, el sujeto no puede nunca coincidir con el "yo". Análogamente, se puede afirmar que el inconsciente es el sujeto ignorado por el "yo".

Ya hemos expresado antes (10) que la aparición de la "segunda tópica" produjo un júbilo entre los analistas, pues era la forma de recuperar el "querido y ansiado yo", pero lo que Freud quería mostrar en realidad era justo lo contrario: recordarnos que entre el sujeto del inconsciente y la organización del "yo", no solo hay disimetría absoluta, sino que hay diferencia radical. Hablamos aquí de "descentramiento" ya que el núcleo de nuestro ser no puede coincidir con el ego. 
ORIGINALES Y REVISIONES

Esto se demuestra cuando escuchamos al paciente y contemplamos que se quiere ver en el "yo" y hasta se cree que es el "yo", pero no es ese el lugar que le corresponde; su sitio se encuentra más allá. La mayor parte de aquello sobre lo que el sujeto cree poseer una certeza reflexiva no es más que una disposición superficial, racionalizada, justificada secundariamente de lo que es su propio ser, que descansa, como luego veremos, en su deseo.

Añadimos que el sujeto cuando habla no sabe lo que dice porque no se ve realmente, solo acierta a contemplarse del lado del ego y escucha tan solo aquello que proviene de este último, por propia boca o por la boca del "otro" (nota 5). De ahí la necesidad de un analista que sirva de testigo para esas palabras que se pronuncian y no son recogidas por quién las enuncia.

El sujeto se representa con la letra (S).

2.5. El Otro. Lacan diferencia con gran claridad el "otro", representado como (a'), y el "Otro", para el que utiliza el matema (A). El gran "Otro" no es ese "otro" que es intercambiable con el "yo", del que es su imagen y que hemos estudiado líneas arriba; se trata de ese otro sujeto que se constituye en la "alteridad fundamental", el "Otro radical" podríamos decir. Con este último no puede haber identidad alguna y trasciende todo lo ilusorio, lo imaginario. Se encuentra, por tanto, más allá del "muro del lenguaje".

El Otro se halla inscrito en el "registro simbólico", el lugar en el que está constituida la palabra y que es desconocido por el "yo". En este sentido, se tiene la ilusión de que la palabra procede del "yo", pero no es así pues se organiza en el Otro; de ahí que Lacan lo nombre como: "tesoro de significantes".

Indica esto que la palabra se encuentra más allá del propio control consciente, viene desde "otro lugar", fuera de la conciencia.

El lenguaje precede a nuestro nacimiento, es independiente de nosotros, no nos pertenece específicamente. A partir del momento en que venimos al mundo van a producirse una serie de palabras (significantes) que nos van a ubicar en la estructura familiar e incluso social. Estas palabras (que proceden del Otro) son fundadoras del sujeto y, al mismo tiempo, lo apresan, forma lo simbólico que lo atrapa y que el análisis pacientemente debe descubrir (ver figura 2).

2.6. La relación “Otro-Sujeto". Esta conexión implica recibir del Otro el lugar que le corresponde a uno en el tejido social, lo que supone como hemos visto, la realización simbólica del sujeto. De ahí, que nos encontremos en el "eje simbólico".

Aquí hallamos en Lacan ecos levi-straussianos (5) ya que se entiende la malla social como una organización de lenguaje en la que se encuentran ciertos "lugares" en los que el sujeto es ubicado desde los designios del Otro, de quién viene la "verdadera palabra". En este sentido, un paciente nuestro desde pequeño fue nombrado como el "inteligente de la familia" y el que "irá a la universidad", mientras su hermano era el "torpe", que "solo podrá dedicarse a trabajos que no requieran esfuerzo intelectual"; 
tales designios procedentes de la alteridad radical se cumplieron a rajatabla en ambos hermanos. Nuestro paciente, a quién no le gustaba estudiar, tubo que seguir los pasos marcados por ese gran Otro; su análisis personal desvela lo que serían para él verdaderas inclinaciones profesionales más allá de aquel lugar simbólico en el que había sido coagulado.

De lo expuesto se sigue que la relación $(A->$ S) representa al inconsciente, por eso Lacan afirma que "el inconsciente es el discurso del Otro". Pero, el inconsciente no es fácil de conocer, lo que se debe a que su discurso tiene que pasar por la mediación de los sustratos imaginarios ("yo" y "otro"), que se interponen como un obstáculo pétreo al conocimiento (ver figura 3)

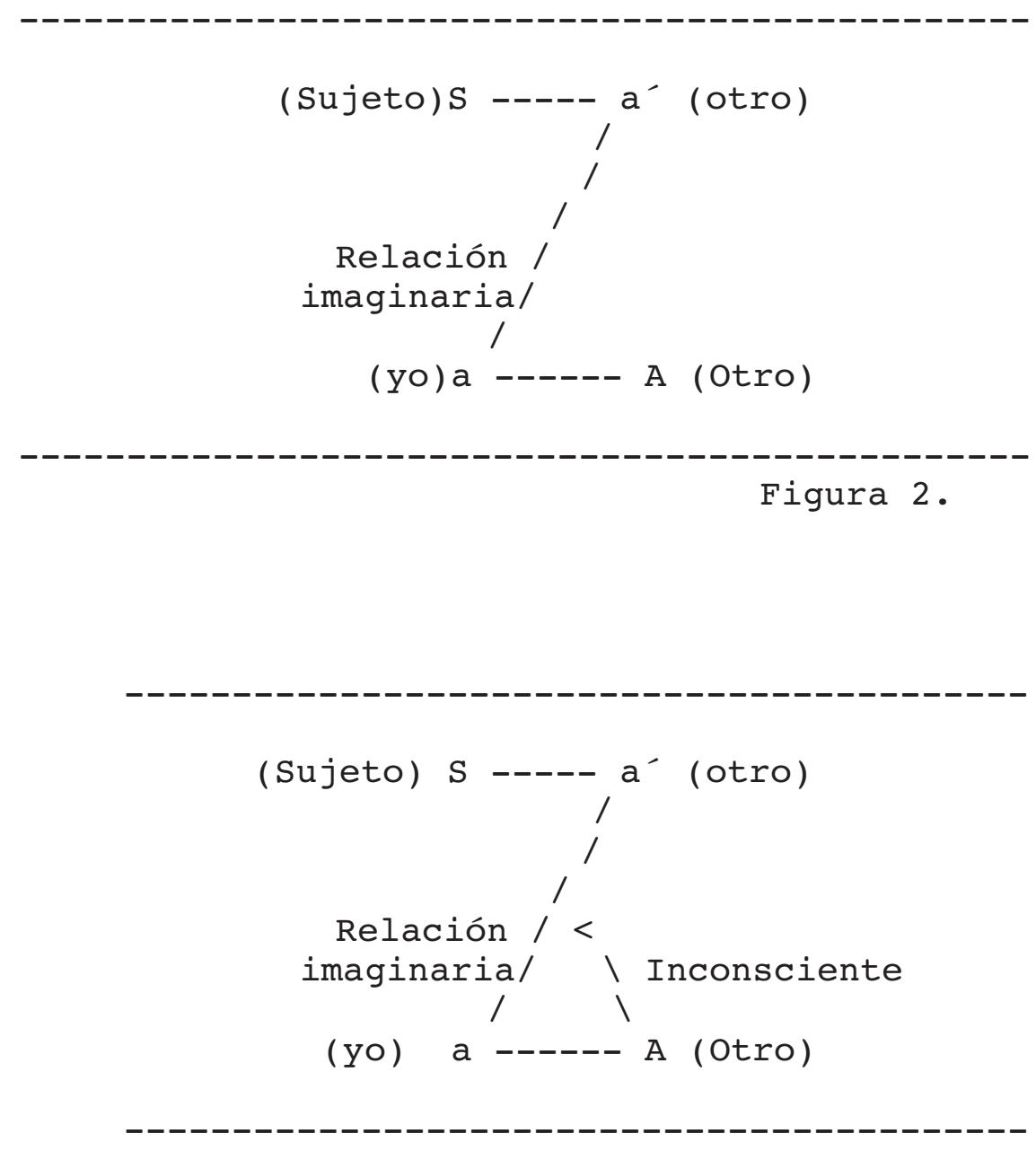

Figura 3 . 
Lacan utiliza el "esquema L" (entre otras cosas) para colocar la palabra en la dimensión intersubjetiva, pero existe algo que parece paradójico en toda esta trama: el sujeto se halla separado de sí mismo por el orden del lenguaje y, paralelamente, se construye como sujeto por el lenguaje ¿cómo puede ser esto? Lacan, apoyándose en Saussure (16), diferencia entre "palabra" y "lenguaje" indicando que mientras la función de la primera es simbólica, el uso del lenguaje es imaginario. Este último adquiere la función imaginaria en tanto objetiviza al sujeto como "yo", digamos que lo clave en una "representación-imagen" de la que no puede liberarse en lo sucesivo. La palabra, en cambio, cumple la función de reconocimiento subjetivo oponiéndose a la función objetivante del lenguaje (17).

Notas.

1. El espacio topológico, como estamos viendo, no implica medición alguna y eso lo hace apto para abordar diferentes aspectos de la vida mental, lo que puede comprobarse fácilmente en buena parte de nuestros pacientes. Los ejemplos que podemos poner son numerosos, pero utilizaremos uno en concreto: se trata de una paciente originaria de Valladolid y muy dependiente de su madre que se viene a vivir a Sevilla debido a su reciente matrimonio; pues bien, a pesar de la distancia entre aquella ciudad y la nuestra, continúa la dependencia materna como si su progenitora se encontrase a su lado, hallándose en realidad a cientos de kilómetros: la llama todos los días por teléfono y se lleva una hora diaria hablando con ella. Como puede verse, no importa la distancia física con su madre, sino la relación que mantiene con ella.

2. Quizás convenga aquí recordar la diferencia entre "aritmética" y "álgebra". Mientras la primera utiliza números, la segunda implica el uso de letras sustituyendo a estos últimos, lo que permite introducir incógnitas $(\mathrm{x}, \mathrm{y}, \mathrm{z}, \ldots)$ en el razonamiento matemático. De ahí que el álgebra sea una generalización de la aritmética, lo que ha permitido entender las "estructuras" en matemática, con las importantísimas ventajas que ello ha supuesto en el dominio de las ciencias exactas.

3. Este punto se confunde frecuentemente con la genética, dado que se dice coloquialmente: "fulano es como su abuelo" y "tiene sus mismos genes". El análisis demuestra que el "yo", en el proceso de identificación, se apropia de las características del semejante, identificándose con él. Se puede observar, además, que las identificaciones muchas veces son contradictorias entre sí. Este es el caso de un paciente que atendimos, identificado con un padre débil y apocado, que logra salir de ese aspecto que él siente como "inhibición" o "bloqueo" mediante una identificación contraria a la anterior, con un tío materno, hombre decido y capaz, a quién su madre tenía en gran estima y consideración. Nuestro consultante puede contemplar en sí mismo la 
contradicción, que se pone de manifiesto en distintas situaciones en las que no puede salir del apocamiento que le embarga, mientras que en otras se descubre decidido y afrontándolas con gran entereza.

4. Lo aquí expuesto puede constatarse fácilmente en las relaciones convencionales. Pongamos un ejemplo al respecto para obtener una mayor claridad expositiva: se trata de un conocido programa de radio que tiene una gran audiencia, una de cuyas ediciones comenzaba de la siguiente forma (nombramos con distintas letras a los asistentes al mismo):

-Locutora: Bienvenidos a nuestro programa. Hoy contamos con una serie de ilustres invitados, a quiénes recibimos calurosamente. Hola A., te veo guapísima, no sé chica lo que te haces para estar tan atractiva.

-A: Hola O. Tú sí que estás encantadora y el programa que diriges es el más interesante de cuántos hay actualmente en la radio.

-Locutora: Gracias A., de todo corazón. Presentamos a continuación a nuestro segundo invitado. Hola B., desde luego por ti no pasan los años, estás fabuloso.

-B: Gracias O., estoy encantado de estar aquí contigo, porque creo que sabes llevar las riendas como nadie y le das tu toque personal a cuánto haces. Te felicito.

-Locutora: muchas gracias y encantada de tenerte aquí. El tercer invitado viene de lejos y ha tenido la gentileza de acudir a nuestra llamada. Hola guapísima, sabes que estamos muy felices de tenerte aquí....

Etc., etc.

Se puede observar, con toda claridad, el narcisismo y el encumbramiento del "yo" que se ponen en escena, aunque este caso es un ejemplo donde los aspectos que estudiamos se muestran de un modo exagerado, debido a las características del programa y al tema del que se ocupa.

5. Lacan subraya que: "El sujeto no sabe lo que dice, y con toda razón, puesto que no sabe lo que él es".

BIBLIOGRAFÍA:

(1) Lacan J. Seminario 2: El yo en la Teoría de Freud y en la Técnica Psicoanalítica. Barcelona: Paidós, 1986.

(2) Thines G.; Lempereur A. Diccionario general de ciencias humanas. Madrid: Cátedra, 1978.

(3) García Arroyo J. M. Jacques Lacan: una biografía intelectual (Parte III). Anales de Psiquiatría 2005, 21 (2), 67-72.

(4) Lacan J. Seminario 4: La relación de objeto. Barcelona: Paidós, 1994.

(5) LÉvi-Strauss C. Las estructuras elementales del parentesco (2 vols.). Barcelona: PlanetaAgostini, 1985. 
(6) Lévi-Strauss C. Antropología estructural. Mexico: Siglo XXI, 1997.

(7) FREUD S. Análisis fragmentario de una histeria (“caso Dora”); en Obras Completas (vol. 1). Madrid: Biblioteca Nueva, 1981.

(8) FreUd S. Sobre la psicogénesis de un caso de homosexualidad femenina; en Obras Completas (vol. 3). Madrid: Biblioteca Nueva, 1981.

(9) LaCAn J. Seminario 5: Las formaciones del inconsciente. Barcelona: Paidós, 1999.

(10) García Arroyo J. M.; Domínguez López M. L. El concepto del “yo" en el corpus freudolacaniano y sus consecuencias en la práctica clínica y psicoterapéutica. Anales de Psiquiatría 2008, 24 (3).

(11) LaCAN J. El estadio del espejo como formador de la función del yo tal como se nos revela en la experiencia psicoanalítica; en Escritos (vol. 1). Madrid: Siglo XXI, 1977.

(12) García Arroyo J. M. ¿En qué consiste el estadio del espejo? Anales de Psiquiatría 2005, 21 (3), 102-10.

(13) Hartmann H. Ensayos sobre la psicología del yo. Mexico: FCE, 1969.

(14) Freud A. El yo y los mecanismos de defensa. Barcelona: Planeta-Agostini, 1984.

(15) Lacan J. Seminario 1: Los escritos técnicos de Freud. Barcelona: Paidós, 1981.

(16) De Saussure F. Curso de lingüística general. Madrid: Alianza, 1990.

(17) Eidelsztein A. Modelos, esquemas y grafos en la enseñanza de Lacan. Buenos Aires: Manantial, 1992. 